\title{
Rapamycin Alleviates Cancellous Bone Loss and Cortical Bone Porosity in Ovariectomized Mice but Not Overall Biomechanical Performance
}

\section{Ruoyao Li}

Southern Medical University Nanfang Hospital

Junhao Liu

Southern Medical University Nanfang Hospital

\section{Zhiping Huang}

Southern Medical University Nanfang Hospital

\section{Xiuhua Wu}

Southern Medical University Nanfang Hospital

\section{Zhou Yang}

Southern Medical University Nanfang Hospital

Junyu Lin

Southern Medical University Nanfang Hospital

Qi Liu

Southern Medical University Nanfang Hospital

\section{Zucheng Huang}

Southern Medical University Nanfang Hospital

Qingan Zhu ( $\nabla$ qinganzhu@gmail.com )

Southern Medical University Nanfang Hospital https://orcid.org/0000-0002-0412-0515

\section{Research article}

Keywords: Rapamycin, Ovariectomy, Cortical bone, Cancellous bone, Biomechanics, Long bones

Posted Date: September 21st, 2021

DOI: https://doi.org/10.21203/rs.3.rs-895657/v1

License: (c) (i) This work is licensed under a Creative Commons Attribution 4.0 International License. Read Full License 


\section{Abstract}

Background: Recent studies have shown that rapamycin (Rapa) rescues cancellous bone loss of osteoporosis models, but its effects on cortical bone and the biomechanical properties remain uncertain. This study was aimed to determine whether rapamycin improve the quality of long bones in ovariectomy (OVX)-induced osteoporosis.

Methods: Thirty female C57BL/6J mice were randomly divided into Sham, OVX and OVX+Rapa group. Mice in the OVX+Rapa group were injected intraperitoneally with $1.5 \mathrm{mg} / \mathrm{kg}$ rapamycin daily after ovariectomy. After 12 weeks, the microstructures of femurs and the vascular canal porosity tibiae were analyzed by micro-CT. The compressive stiffness of the distal femurs was calculated with the micro-finite element method, and the bending strength of the tibiae was evaluated with a three-point bending test. Western blot of LC3, P62 was used to assess autophagy activity of bone. The number of osteoclasts was quantified by tartrate-resistant acid phosphatase (TRAP) staining. ELISA detected the concentration of bone-specific alkaline phosphatase (BALP) in serum.

Results: OVX led to a decrease in cross-sectional areas of the mid-diaphyses and distal femoral cortical bones, increasing the cortical bone vascular canal porosity from $1.42 \%$ to $4.79 \%$. The rapamycin reduced cancellous bone loss and decreased cortical bone vascular canal porosity by $3.9 \%$, but further reduced the thickness of the distal femoral cortical bone by $16.7 \%$, with no significant effect on the cortical bone in the mid-diaphyses. Compared to the Sham group, OVX mice showed a decrease in distal femoral stiffness to $6497 \mathrm{~N} / \mathrm{mm}$ and a decrease in tibial maximum load to $6.08 \mathrm{~N}$, while rapamycin intervention did not significantly improve the decreased biomechanical properties. Moreover, rapamycin remarkably reduced the TRAP-positive osteoclasts and the concentration of serum BALP by $80.6 \%$ and $30.8 \%$, respectively. Autophagy was activated in the OVX group compared with the sham group.

Conclusions: This study has demonstrated that rapamycin ameliorates cancellous bone loss and cortical bone porosity in ovariectomized mice but partly reduces the size of cortical bone, while has no effect on improving the biomechanical performance. Additionally, the present study also proved that OVX led to both cancellous and cortical bone loss, with attenuated biomechanical properties of long bones.

\section{Introduction}

Rapamycin (Rapa), also known as sirolimus, is a macrolide compound initially discovered in a soil sample from Easter Island. Rapamycin is widely used in the clinic as an immunosuppressor, especially for preventing rejection of kidney transplants. Growing interest is attracted by rapamycin nowadays in treatment for certain diseases such as cancer, tuberous sclerosis complex, lymphangioleiomyomatosis and neurological diseases [11]. Rapamycin also functions as a specific inhibitor of the mammalian target of rapamycin complex 1 (mTORC1) and effectively activates autophagy [25], which is an evolutionarily conserved process in eukaryotes that degrades damaged proteins and organelles [10], also identified as type $₫$ programmed cell death. 
Researches have demonstrated that rapamycin has a significant effect on rescuing cancellous bone loss by activating autophagy in osteoporosis animal models, including senile rats [16], chronic high dose alcohol-treated mice [15] and ovariectomized (OVX) mice [18]. However, rapamycin may not have the protective effect on cortical bone, Wu et al. indicated that rapamycin increases cancellous bone mass in OVX mice with iron accumulation, but it makes no difference to cortical bone [23]. Moreover, in the study for OVX mice treated with everolimus, the second novel rapamycin analogue, Kneissel et al. suggested similar results [9]. Taken together, rapamycin provides protection to cancellous bone, but the protection to the cortical bone has not been observed. It is also unclear whether treatment with rapamycin improves the mechanical properties of long bones, especially in OVX mice. Therefore, effects of rapamycin on cortical bone of OVX mice and on the quality of long bone as a whole deserve further study.

In addition, it is generally accepted that OVX results in cancellous bone loss. However, whether cortical bone loss or attenuated biomechanical properties occur in OVX mice is still controversial[1, 3, 17, 19-21]. Sharma et al. suggested that increased vascular canal porosity in the cortical bone of OVX mice could be detected by high-resolution micro-CT[21]. Therefore, it is necessary to study the changes in cortical bone and biomechanical properties of mice after the long-term effect of OVX.

The primary objective of the present study was to evaluate the effect of rapamycin treatment on microstructure of cancellous and cortical bones in ovariectomy (OVX)-induced osteoporosis, and the overall biomechanical performance of long bones. The secondary objective was to investigate the effects of OVX on the cortical bone and biomechanical properties of long bones.

\section{Methods}

\section{Study Design}

Thirty female C57BL/6J mice were purchased from the Laboratory Animal Centre of Southern Medical University. After two weeks of adaptive feeding, the 8-week-old mice with an average weight of $18.17 \mathrm{~g}$ were randomly divided into three groups (ten in each group) including Sham group, OVX group and OVX+Rapa group. Mice in OVX group and OVX+Rapa group received bilateral ovariectomy under general anesthesia by isoflurane. For activation of autophagy, a dose of $1.5 \mathrm{mg} / \mathrm{kg}$ Rapa (Solarbio Science \& Technology Co., Ltd, Beijing, China) was administered daily by intraperitoneal injection to mice in OVX+Rapa group. All mice were housed under $12 \mathrm{~h}$ light-dark cycles with free access to food and water, and at the temperature of $23 \pm 1{ }^{\circ} \mathrm{C}$ and humidity of $50 \%$. Weighed and recorded the body weight of mice every two weeks. After feeding for 12 weeks, mice were anesthetized by isoflurane, and then femurs and tibias were harvested after sacrifice and fixed in $4 \%$ paraformaldehyde or frozen at $-80^{\circ} \mathrm{C}$ directly. Seven mice in each group were selected for micro-CT scanning on the same side of the femurs, the other side of the femurs for histological staining, and the remaining femurs for Western blot analysis. In addition, the ipsilateral tibia of each group of seven mice was used for the three-point bending test, and one tibia was used for high-resolution micro-CT scanning. 


\section{Micro-CT Scanning and Analysis}

Femurs were scanned by the micro-CT system ( $\mu$ CT 80, Scanco Medical, AG, Switzerland) with an isotropic voxel size of $12 \mu \mathrm{m}$, and at a voltage of $55 \mathrm{kV}$ and a current of $145 \mu \mathrm{A}$. Referring to our previous study, distal femurs and mid-diaphyses of femurs were defined as the region of interest for analysis [14]. The parameters of cancellous bone included bone volume/tissue volume (BV/TV), connection density of trabecular (Conn.D), trabecular number (Tb.N), trabecular thickness (Tb.Th), trabecular separation (Tb.Sp) and tissue mineral density (TMD), while the cortical bones from both distal femurs and middiaphyses of femurs were assessed by cross-sectional bone area (Barea), total cross-sectional area (Tarea), cross-sectional bone area/total cross-sectional area (Barea/Tarea), cortical thickness (Ct.Th) and tissue mineral density (TMD).

Besides, tibiae were scanned by the high-resolution micro-CT system (MultiScale Voxel-1000, Sanying Precision Instruments Co., Tianjin, China) at a resolution of $2.48 \mu \mathrm{m}$, and at a voltage of $60 \mathrm{kV}$ and a current of $40 \mu \mathrm{A}$. The region of interest was defined as the starting $1 \mathrm{~mm}$ from the most distal end of the growth plate in proximal tibial. The porosity, diameter and volume of vascular canal were calculated.

\section{Simulated Compressive Test by the Micro-Finite Element}

In order to assess the biomechanical properties, distal femoral models were established by highresolution bone microstructure images. Built on the three-dimensional structures, the simulated compressive test was conducted using micro-finite element analysis (SCANCO Medical AG, Version 1.13) [14]. Axial compressive test in the elastic range was simulated by homogeneous and isotropic elastic materials. Parameters of the materials were Poisson's ratio $v=0.3$ and Youngs modulus $E=10,000 \mathrm{MPa}$. The tests were conducted by "z-directional high friction compressive test", i.e. along the femoral longitudinal direction to evaluate the compressive stiffness of femoral bone.

\section{Three-Point Bending Test}

Reference to the previous study [24], biomechanical properties of tibiae were evaluated by three-point bending test with materials testing machine (Electropuls E1000, Instron Inc., MA, USA). After being thawed at room temperature for $1 \mathrm{~h}$, tibiae were placed on two supports with a span of $10 \mathrm{~mm}$ on a slider (Fig. 1). A compressive force was applied to the sample at a constant speed of $2 \mathrm{~mm} / \mathrm{min}$ until it was broken. The maximum load was determined by the displacement-load curves.

\section{Histological Evaluation and Histochemical Staining}

Femurs were decalcified by 15\% EDTA for 4 weeks and then embedded in paraffin. The samples were cut into sections of $4 \mu \mathrm{m}$ in thickness and followed by being deparaffined in xylene and rehydrated by graded 
concentration of ethanol. To observe the morphologic changes of trabecular and the presence of osteoclasts, hematoxylin-eosin (HE) staining (Fude Biological Technology Co., Ltd, Hangzhou, China) and tartrate-resistant acid phosphatase (TRAP) staining (Nanjing Jiancheng Bioengineering Institute, Nanjing, China) were performed as described by manufacturers' protocols, respectively. Numbers of TRAP-positive osteoclasts were counted with Image J 1.52 software (National Institutes of Health, USA) for semiquantitative analysis. The area from the growth plate to epiphysis was defined as the region of interest.

\section{Western Blot Analysis}

Intact femurs were frozen in liquid nitrogen and lysed in RIPA buffer (Beyotime, Shanghai, China) containing PMSF, protease inhibitor and protein phosphatase inhibitor. After being ground in tissue homogenization, samples were centrifuged at $4^{\circ} \mathrm{C}$ and then the supernatant was collected. Lysates were added with loading buffer and boiled at $100^{\circ} \mathrm{C}$ for $5 \mathrm{~min}$, saved at $-80^{\circ} \mathrm{C}$ for Western blot analysis. According to the molecular weight, proteins were separated by $12 \%$ or $15 \%$ SDS-PAGE gels and transferred to PVDF membranes. The membranes were blocked in $5 \%$ BSA for $1 \mathrm{~h}$ and followed by incubating with primary antibodies against LC3B (1:500, Cell Signaling Technology, USA), P62 (1:1000, Cell Signaling Technology, USA) or GAPDH (1:10000, abcam, UK) at $4^{\circ} \mathrm{C}$ overnight. After three washes in TBST (5 min each time), membranes were incubated with secondary antibodies (1:5000, Beyotime, China) for $1 \mathrm{~h}$ at room temperature. The signal was finally determined by enhanced chemiluminescence (ECL) system and quantified by Image J 1.52 software (National Institutes of Health, USA).

\section{ELISA Assay}

The level of bone-specific alkaline phosphatase (BALP) in serum was detected using BALP ELISA kit (CUSABIO, E11914m, US). The procedure was performed according to the introductions of manufacture.

\section{Statistical Analysis}

Statistical analysis was performed by SPSS v.23.0 software. One-way analysis of variance was used to analyze the difference between the whole three groups. Multiple comparisons were analyzed by the Student-Newman-Keuls test. $P \otimes 0.05$ was considered statistically significant.

\section{Results}

\section{Body Weight}

Because the mice were not born in the same litter, the initial body weight of the mice in the OVX+Rapa group was lower than that of the other two groups (Fig. 2). To exclude the influence of body weight on the 
results, data were adjusted for initial body weight. After 12 weeks of feeding, there was no significant difference in body weight between three groups.

\section{Micro-CT Measurements of Cancellous and Cortical Bone}

Micro-CT was used to determine the effects of ovariectomy and/or rapamycin on the bone. The data of micro-CT show that significant bone loss was occurred both in cancellous bone and cortical bone due to ovariectomy (Fig. 3, Fig. 4, Table 1). Of note, the cross-sectional cortical bone areas, recorded as Barea, of both mid-diaphyses and the distal femurs in OVX mice were lower than that of the sham group (the OVX group vs. the Sham group: $0.71 \mathrm{~mm}^{2}$ and $0.73 \mathrm{~mm}^{2}$ vs. $0.78 \mathrm{~mm}^{2}$ and $0.81 \mathrm{~mm}^{2}$ in the mid-diaphyses and distal femurs, respectively, both $P \otimes 0.05$, Table 1).

Table 1

Micro-CT analysis of cortical bone from distal end and mid-diaphyses of femurs

\begin{tabular}{|c|c|c|c|c|c|c|}
\hline Segments & Groups & $\begin{array}{l}\text { Barea } \\
\left(\mathrm{mm}^{2}\right)\end{array}$ & $\begin{array}{l}\text { Tarea } \\
\left(\mathrm{mm}^{2}\right)\end{array}$ & BA/TA & Ct.Th (mm) & $\begin{array}{l}\text { TMD (mg } \\
\mathrm{HA} / \mathrm{ccm})\end{array}$ \\
\hline \multirow{3}{*}{$\begin{array}{l}\text { Mid- } \\
\text { diaphyses }\end{array}$} & Sham & $0.78 \pm 0.03$ & $1.76 \pm 0.06$ & $0.44 \pm 0.02$ & $0.18 \pm 0.01$ & $967 \pm 19$ \\
\hline & OVX & $0.71 \pm 0.03^{a}$ & $1.72 \pm 0.08$ & $0.42 \pm 0.01^{a}$ & $0.17 \pm 0.00^{a}$ & $955 \pm 17$ \\
\hline & $\begin{array}{l}\text { OVX + } \\
\text { Rapa }\end{array}$ & $0.71 \pm 0.03^{a}$ & $1.81 \pm 0.04$ & $0.43 \pm 0.01^{b}$ & $0.17 \pm 0.01^{\mathrm{a}}$ & $1020 \pm 31^{a, b}$ \\
\hline \multirow[t]{3}{*}{ Distal end } & Sham & $0.81 \pm 0.09$ & $0.95 \pm 0.09$ & $0.85 \pm 0.06$ & $0.12 \pm 0.01$ & $874 \pm 15$ \\
\hline & OVX & $0.73 \pm 0.04^{a}$ & $\begin{array}{l}0.85 \pm 0.06 \\
a\end{array}$ & $0.86 \pm 0.02$ & $0.12 \pm 0.01$ & $844 \pm 19^{a}$ \\
\hline & $\begin{array}{l}\text { OVX + } \\
\text { Rapa }\end{array}$ & $0.66 \pm 0.04^{a}$ & $0.80 \pm 0.04^{a}$ & $\begin{array}{l}0.92 \pm 0.03^{a}, \\
b\end{array}$ & $\begin{array}{l}0.10 \pm 0.01^{a}, \\
b\end{array}$ & $894 \pm 35^{b}$ \\
\hline $\begin{array}{l}\text { Data are pre } \\
\text { a } P<0.05 \rrbracket \mathrm{co} \\
\text { b } P<0.05 \bigotimes \mathrm{be}\end{array}$ & $\begin{array}{l}\text { pared with } \\
\text { een OVX }\end{array}$ & $\begin{array}{l}\text { am group } \\
\text { OVX + Rapa } \mathrm{S}\end{array}$ & $\begin{array}{l}\text { viation } \\
\text { oup }\end{array}$ & & & \\
\hline
\end{tabular}

As for cancellous bone, the OVX+Rapa group showed significantly higher BV/TV, Conn.D, Tb.N, Tb.Th, $\mathrm{TMD}$ with lower Tb.Sp than that in the OVX group ( $P \otimes 0.05)$. Even comparing with the Sham group, the OVX+Rapa group had striking increases of BV/TV, Conn.D, Tb.Th and TMD (P凶0.05, Fig. 3, Fig. 4).

However, for cortical bone from mid-diaphyses of femurs, Barea and Ct.Th in the OVX+Rapa group were not significantly different from the OVX group ( $P \bowtie 0.05$, Table 1). Furthermore, compared to the OVX group, 
the cortical bone of distal femur in the OVX+Rapa group had a 9.6\% decrease in Barea, a $5.9 \%$ decrease in Tarea, and a $16.7 \%$ significant decrease in Ct.Th (the OVX group vs. the OVX+Rapa group: $0.12 \mathrm{~mm}$ vs. $0.10 \mathrm{~mm}, P \otimes 0.05$, Fig. 3, Table 1).

Moreover, as shown in Fig. 5, high-resolution micro-CT scans of the proximal tibia indicated that the vascular canal porosity in the OVX group was higher than that of the Sham group (the OVX group: 4.79\%, the Sham group: $1.42 \%$ ), while the porosity in the OVX+Rapa group was reduced to $0.89 \%$. Both in the Sham group and the $O V X+R a p a$ group, canals with diameters less than $5 \mu \mathrm{m}$ were the most in number, while those with diameters greater than $80 \mu \mathrm{m}$ accounted for the largest volume, at $43.76 \%$ and $28.20 \%$ respectively. In the OVX group, the number of canals with a diameter greater than $100 \mu \mathrm{m}$ was the largest and accounts for $70.37 \%$ of the volume of the canals.

\section{Biomechanical Properties of Long Bones}

In a simulated compressive test by the micro-finite element, the data show that the stiffness of the distal femur in the OVX group and OVX+Rapa group were declined considerably to $6497 \pm 376 \mathrm{~N} / \mathrm{mm}$ and $6098 \pm 357 \mathrm{~N} / \mathrm{mm}$ (Fig. 6A, both $P \otimes 0.05$ ) respectively, compared to the stiffness in the Sham group $(7414 \pm 732 \mathrm{~N} / \mathrm{mm})$. Compared with the Sham group, the stiffness of distal femurs in the OVX group was decreased by about $12.0 \%$ while the treatment of rapamycin made a further decrease of $6.1 \%$.

Similarly, the three-point bending test of the tibiae showed a sharp decline by $24.8 \%$ in maximum load in the OVX group $(6.08 \pm 0.91 \mathrm{~N})$ compared to the Sham group $(8.08 \pm 1.04 \mathrm{~N}$, Fig. 6B, $P \otimes 0.05)$. Moreover, rapamycin intervention $(6.35 \pm 0.77 \mathrm{~N})$ could not significantly increase the maximum load reduced by ovariectomy (Fig. 6B, P凶0.05).

\section{Histological Characteristics and Bone Metabolism}

The morphology of cancellous bone in distal femurs was observed by HE staining. As shown in Fig. 7A-C, compared with the Sham group, the number of trabeculae in the OVX group reduced and the separation increased, while the microstructure destruction in the OVX+Rapa group was alleviated.

To determine whether the process of autophagy is affected by ovariectomy, proteins extracted from

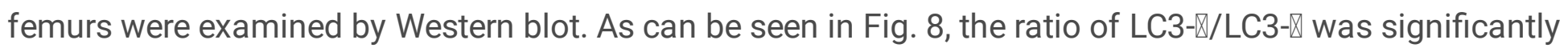
higher in OVX mice, with obviously lower expression of P62 (Pख0.05).

TRAP staining was performed to investigate how rapamycin act on osteoclasts in cancellous bone. In comparison with the Sham group, the TRAP-positive osteoclasts in the OVX group were obviously increased, while the rapamycin treatment reduced it dramatically ( $P \otimes 0.05$, data not shown, Fig. 7D-F).

ELISA was performed to detect the BALP concentration in serum as an indicator of osteoblast differentiation. Compare with the Sham group $(314.7 \mathrm{ng} / \mathrm{mL})$ and the OVX group (303.9 $\mathrm{ng} / \mathrm{mL})$, BALP 
concentration of the OVX+Rapa group was significantly reduced $(210.2 \mathrm{ng} / \mathrm{mL}, P \otimes 0.05)$.

\section{Discussion}

Researches have shown that rapamycin has an advantageous impact on cancellous bone, but its effect on cortical bone or overall biomechanical properties of long bones is rarely reported $[9,15,16,18,23]$. The present study verified that rapamycin improved cancellous bone at the distal end of the femur, but we also found a reduction in cortical bone of long bones. We further confirmed that rapamycin did not ameliorate the biomechanical properties reduced by ovariectomy, either in the distal femur or in the tibia. This may be related to the excessive autophagy caused by rapamycin and ovariectomy combined. In addition, this study also confirmed that ovariectomy resulted in cortical bone loss in mice, a phenomenon not observed in some studies of OVX induced osteoporosis, which may be related to the long-term observation of OVX mice.

Studies have shown that rapamycin has a significant effect on reducing cancellous bone loss in osteoporotic animal models, including age-related osteoporosis, alcohol-induced osteoporosis, and postmenopausal osteoporosis $[15,16,18]$. The present study has also confirmed this effect of rapamycin in OVX mice. However, as the treatment strategy of osteoporosis is mainly focused on cancellous bone, there is still a lack of attention on the effect of cortical bone. A study by Wu et al. indicated that in OVX mice with iron accumulation, rapamycin has no effect on cortical bone although it increases cancellous bone mass [23]. Kneissel et al. suggested similar results in the study of OVX mice treated with rapamycin analogue [9]. In the present study, we evaluated the cortical bone not only at the diaphyses, but also at the distal femur as the same level of the cancellous bone. The results have shown that rapamycin has no effect on cortical bone in mid-diaphyses, as had been found in previous studies. However, we did observe further reductions in the area and thickness of the cortical bone in the distal femur. This suggests that contrary to the superior effects on cancellous bone, rapamycin treatment could not alleviate cortical bone loss in OVX mice. In addition, it also implies that the cortical bone from distal femurs responds more rapidly to the intervention of rapamycin than that from mid-diaphyses.

Interestingly, although rapamycin further decreases the bone area and thickness of cortical bone at the end of long bones, it reduced the vascular canal porosity in this section. This suggests that rapamycin reduces the size of the cortical bone but improves its density. The effects of rapamycin on bone tissue are therefore complex.

Since cancellous and cortical bone showed opposite trends under rapamycin treatment, it is necessary to assess the effect of rapamycin on long bone in terms of overall biomechanical function. The results indicate that rapamycin did not improve the biomechanical properties of the long bones of OVX mice, either in terms of stiffness or maximum load. Here, the simulated compressive test was performed on the distal femurs only. For mid-diaphyses of femurs, the biomechanical properties are mainly determined by the cross-sectional bone area, showing no change after the rapamycin treatment. 
It is worth mentioning that whether cortical bone loss and attenuated biomechanical properties occur in OVX mice remains controversial. The present study has demonstrated that cross-sectional bone area and cortical bone thickness were decreased in the mid-diaphyses of femurs, which is consistent with the investigation of tibial cortical midshafts from OVX mice [19]. Furthermore, reduced cortical bone crosssectional area and bone mineral density in the distal femurs were also observed. However, Pereira et al. suggested that for rats 10 weeks after ovariectomy, cancellous bone was damaged but not cortical bone in the distal femur [17]. It is possible that differences in the timing of intervention as well as species differences are responsible for the different results.

Besides, this study supports that OVX results in higher vascular canal porosity, weakening the biomechanical properties of the long bone, which is in good agreement with the study of femoral necks from rats [1]. A study of rats 10 weeks post-OVX also has indicated that estrogen deficiency leads to increased cortical bone vascular canal porosity, which has been proved to closely relate with bone strength $[3,21]$. Shah et al. verify this change in cortical vascular canal porosity in a study of rats 4 and 8 weeks after ovariectomy. However, they tend to refute the decreased mechanical properties induced by ovariectomy, because the indentation hardness and elastic modulus are unaffected [20]. The reason for the different results may be that the indentation hardness and elastic modulus and the axial compression stiffness and maximum load measured in this study are evaluated from different aspects of biomechanical properties. Moreover, we believe that the biomechanical properties of long bones could be weakened during prolonged estrogen deficiency states. Different duration after the intervention may be another reason for the different results.

Here, autophagy activity was evaluated to explore the possible mechanisms of changes in bone. Autophagy acts to degrade the impaired proteins or organelles to maintain cell homeostasis, it can be activated by cellular stress such as under situations of energy deficiency, starvation or hypoxia [4]. The increased ratio of LC3- $₫ / L C 3-\rrbracket$ and reduced P62 expression serve as indexes of activated autophagic activity $[7,8]$. In this study, 12 weeks after the intervention, the OVX group showed markedly higher activity of autophagy in bone tissue when compared with the Sham group, indicating that ovariectomy has an activating function to autophagy. The results are in agreement with previous studies $[2,28]$. Estrogen withdrawal, increased oxidative stress and up-regulation of microRNA-119a-3p are the potential factors for autophagic activation in the bone of OVX mice $[2,5,22]$. Rapamycin has been shown to inhibit mTORC1 specifically, leading to increased autophagy activity [4]. Therefore, for bone tissue of OVX mice, autophagy was further activated with rapamycin intervention.

Researches have indicated that inhibition of autophagy results in decreased differentiation and bone resorption $[13,26]$. Besides, Gavali et al. reported that estrogen reduces osteoblasts apoptosis and enhances mineralization by promoting autophagy [6]. These findings are supported by Yang et al. [27]. Therefore, it is believed that moderate autophagy is advantageous for the survival or functions of osteoclasts and osteoblasts. However, in this study, after further activating autophagy by rapamycin, TRAP-positive osteoclasts were noticeably reduced in the epiphysis, suggesting the damaged osteoclastogenesis. Also, the expression of BALP was decreased, indicating restricted differentiation of 
osteoblasts. We suppose that activated autophagy might act as a protective reaction after ovariectomy to resist cellular stress, while excessive autophagy could instead cause damage. This idea is supported by researches for autophagy in the motor neuron. In neurodegenerative disease, autophagy may have a neuroprotective effect by degrading aggregated proteins, but excessive autophagy by rapamycin treatment results in mitochondria damage and apoptosis in motor neuron [12, 29]. Therefore, in this study, it could be speculated that both osteoclasts and osteoblasts are damaged by excessive autophagy simultaneously, but the mechanisms underlying the inconsistent changes in cancellous and cortical bone require further study.

There are still some limitations in this study that need to be recognized. Firstly, in this study, mice received prolonged rapamycin treatment immediately after ovariectomy, other dosages and intervention time of rapamycin treatment should be taken into consideration. Secondly, there is a lack of molecular biology testing to verify the hypothesized mechanisms, such as the differentiation of osteoblasts. Thirdly, the number of tibiae on which high-resolution micro-CT scanning was performed was limited and was only used as a preliminary determination. On the other hand, the advantages of this study were the long duration of the intervention and the detection of different parts of the cortical bone. Also, we performed biomechanical tests on long bones as a functional assessment.

Taken together, rapamycin may not improve the quality of long bone in OVX mice. Excessive autophagy activity might be a possible mechanism. These findings may help to provide a comprehensive view of rapamycin treatment. In the clinical use of rapamycin, especially for postmenopausal women, there is a need to pay close attention to the possible adverse changes in cortical bone. In addition, we have demonstrated that cortical bone loss and attenuated biomechanical properties occur in the long bone after long time OVX, besides of cancellous bone loss.

\section{Conclusion}

Here, we have indicated that rapamycin treatment for OVX mice rescues cancellous bone loss and cortical bone porosity but partly reduces the size of cortical bone, while could not ameliorate the decreased biomechanical properties of long bones. Therefore, rapamycin does not improve the overall quality of long bones in OVX mice. The high activity of autophagy might account for the results. Besides, we also provide evidence that long time ovariectomy leads to both cancellous and cortical bone loss and decreased biomechanical properties of the long bone.

\section{Abbreviations}

Rapa, Rapamycin

OVX, Ovariectomy

TRAP, Tartrate-resistant acid phosphatase 
BALP, Bone-specific alkaline phosphatase

mTORC1, Mammalian target of rapamycin complex 1

$\mathrm{BV} / \mathrm{TV}$, Bone volume/tissue volume

Conn.D, Connection density of trabecular

Tb.N, Trabecular number

Tb.Th, Trabecular thickness

Tb.Sp, Trabecular separation

TMD, Tissue mineral density

Barea, Eross-sectional bone area

Tarea, Total cross-sectional area

Barea/Tarea, Cross-sectional bone area/total cross-sectional area

Ct.Th, Cortical thickness

HE, Hematoxylin-eosin

$E C L$, Enhanced chemiluminescence

\section{Declarations}

\section{Ethics approval and consent to participate:}

The animal study was reviewed and approved by the Experimental Animal Welfare and Ethics Committee of Southern Medical University (No. NFYY-2019-53).

\section{Consent for publication:}

Not applicable.

\section{Availability of data and materials:}

The datasets used and/or analysed during the current study are available from the corresponding author on reasonable request. 


\section{Competing interests:}

The authors declare that they have no competing interests.

\section{Funding:}

Not applicable.

\section{Authors' contributions:}

QZ designed the research and revised the manuscript. RL performed the experiments and wrote the manuscript. JL contributed to analysis and manuscript preparation. $\mathrm{ZH}$ helped conduct the biomechanical experiments. XW and ZY helped perform the micro-CT scanning and analysis. JL, QL and $\mathrm{ZH}$ revised the research with constructive discussions.

\section{Acknowledgements:}

We thank Sanying Precision Instruments Co., Tianjin, China, for conducting the high-resolution micro-CT scanning and analysis.

\section{Authors' information (optional):}

Not applicable.

\section{References}

1. Bagi CM, Ammann P, Rizzoli R, Miller SC. Effect of estrogen deficiency on cancellous and cortical bone structure and strength of the femoral neck in rats. Calcif Tissue Int. 1997;61:336-44. doi:10.1007/s002239900344.

2. Chokalingam K, Roforth MM, Nicks KM, McGregor U, Fraser D, Khosla S, Monroe DG. Examination of ER alpha Signaling Pathways in Bone of Mutant Mouse Models Reveals the Importance of EREDependent Signaling. Endocrinology. 2012;153:5325-33. doi:10.1210/en.2012-1721.

3. Currey JD, Shahar R. Cavities in the compact bone in tetrapods and fish and their effect on mechanical properties. J Struct Biol. 2013;183:107-22. doi:10.1016/j.jsb.2013.04.012.

4. Debnath J, Baehrecke EH, Kroemer G. Does autophagy contribute to cell death? Autophagy. 2005;1:66-74. DOI 10.4161/auto.1.2.1738. doi.

5. Fu JY, Hao LY, Tian YW, Liu Y, Gu YJ, Wu JH. miR-199a-3p is involved in estrogen-mediated autophagy through the IGF-1/mTOR pathway in osteocyte-like MLO-Y4 cells. J Cell Physiol. 
2018;233:2292-303. doi:10.1002/jcp.26101.

6. Gavali S, Gupta MK, Daswani B, Wani MR, Sirdeshmukh R, Khatkhatay MI. Estrogen enhances human osteoblast survival and function via promotion of autophagy. Biochim Biophys Acta Mol Cell Res. 2019;1866:1498-507. doi:10.1016/j.bbamcr.2019.06.014.

7. Hale AN, Ledbetter DJ, Gawriluk TR, Rucker EB. Autophagy Regulation and role in development. Autophagy. 2013;9:951-72. doi:10.4161/auto.24273.

8. Kim KH, Lee MS. Autophagy-a key player in cellular and body metabolism. Nat Rev Endocrinol. 2014;10:322-37. doi:10.1038/nrendo.2014.35.

9. Kneissel M, Luong-Nguyen NH, Baptist M, Cortesi R, Zumstein-Mecker S, Kossida S, O'Reilly T, Lane H, Susa M. Everolimus suppresses cancellous bone loss, bone resorption, and cathepsin K expression by osteoclasts. Bone. 2004;35:1144-56. doi:10.1016/j.bone.2004.07.013.

10. Levine B, Kroemer G. Autophagy in the pathogenesis of disease. Cell. 2008;132:27-42. doi:10.1016/j.cell.2007.12.018.

11. Li J, Kim SG, Blenis J. Rapamycin: one drug, many effects. Cell Metab. 2014;19:373-9. doi:10.1016/j.cmet.2014.01.001.

12. Li L, Zhang $X$, Le W. Altered macroautophagy in the spinal cord of SOD1 mutant mice. Autophagy. 2008;4:290-3. doi:10.4161/auto.5524.

13. Lin NY, Chen CW, Kagwiria R, Liang RF, Beyer C, Distler A, Luther J, Engelke K, Schett G, Distler JHW. Inactivation of autophagy ameliorates glucocorticoid-induced and ovariectomy-induced bone loss. Ann Rheum Dis. 2016;75:1203-10. doi:10.1136/annrheumdis-2015-207240.

14. Liu Q, Xu X, Yang Z, Liu Y, Wu X, Huang Z, Liu J, Huang Z, Kong G, Ding J, Li R, Lin J, Zhu Q. Metformin Alleviates the Bone Loss Induced by Ketogenic Diet: An In Vivo Study in Mice. Calcif Tissue Int. 2019;104:59-69. doi:10.1007/s00223-018-0468-3.

15. Liu Y, Kou X, Chen C, Yu W, Su Y, Kim Y, Shi S, Liu Y. Chronic High Dose Alcohol Induces Osteopenia via Activation of mTOR Signaling in Bone Marrow Mesenchymal Stem Cells. Stem Cells. 2016;34:2157-68. doi:10.1002/stem.2392.

16. Luo D, Ren H, Li T, Lian K, Lin D. Rapamycin reduces severity of senile osteoporosis by activating osteocyte autophagy. Osteoporosis Int. 2016;27:1093-101. doi:10.1007/s00198-015-3325-5.

17. Pereira C, Guede D, Duraes C, Brandao I, Silva N, Passos E, Bernardes M, Monteiro R, Martins MJ. Differential Modulation of Cancellous and Cortical Distal Femur by Fructose and Natural MineralRich Water Consumption in Ovariectomized Female Sprague Dawley Rats. Nutrients. 2019;11. doi: ARTN 2316. 3390/nu11102316.

18. Qi M, Zhang L, Ma Y, Shuai Y, Li L, Luo K, Liu W, Jin Y. Autophagy Maintains the Function of Bone Marrow Mesenchymal Stem Cells to Prevent Estrogen Deficiency-Induced Osteoporosis. Theranostics. 2017;7:4498-516. doi:10.7150/thno.17949.

19. Roberts BC, Giorgi M, Oliviero S, Wang N, Boudiffa M, Dall'Ara E. The longitudinal effects of ovariectomy on the morphometric, densitometric and mechanical properties in the murine tibia: A comparison between two mouse strains. Bone. 2019;127:260-70. doi:10.1016/j.bone.2019.06.024. 
20. Shah FA, Stoica A, Cardemil C, Palmquist A. Multiscale characterization of cortical bone composition, microstructure, and nanomechanical properties in experimentally induced osteoporosis. J Biomed Mater Res A. 2018;106:997-1007. doi:10.1002/jbm.a.36294.

21. Sharma D, Larriera Al, Palacio-Mancheno PE, Gatti V, Fritton JC, Bromage TG, Cardoso L, Doty SB, Fritton SP. The effects of estrogen deficiency on cortical bone microporosity and mineralization. Bone. 2018;110:1-10. doi:10.1016/j.bone.2018.01.019.

22. Wang KK, Niu JL, Kim H, Kolattukudy PE. Osteoclast precursor differentiation by MCPIP via oxidative stress, endoplasmic reticulum stress, and autophagy. J Mol Cell Biol. 2011;3:360-8. doi:10.1093/jmcb/mjr021.

23. Wu J, Wang A, Wang X, Li G, Jia P, Shen G, Chen B, Yuan Y, Zhang H, Yang F, Xu Y. Rapamycin improves bone mass in high-turnover osteoporosis with iron accumulation through positive effects on osteogenesis and angiogenesis. Bone. 2019;121:16-28. doi:10.1016/j.bone.2018.12.019.

24. Wu XH, Huang ZC, Wang XM, Fu ZZ, Liu JH, Huang ZP, Kong GG, Xu XL, Ding JY, Zhu QG. Ketogenic Diet Compromises Both Cancellous and Cortical Bone Mass in Mice. Calcified Tissue Int. 2017;101:412-21. doi:10.1007/s00223-017-0292-1.

25. Wullschleger S, Loewith R, Hall MN. TOR signaling in growth and metabolism. Cell. 2006;124:47184. doi:10.1016/j.cell.2006.01.016.

26. Xiu Y, Xu H, Zhao C, Li JB, Morita Y, Yao ZQ, Xing LP, Boyce BF. Chloroquine reduces osteoclastogenesis in murine osteoporosis by preventing TRAF3 degradation. J Clin Invest. 2014;124:297-310. doi:10.1172/Jci66947.

27. Yang YH, Chen K, Li B, Chen JW, Zheng XF, Wang YR, Jiang SD, Jiang LS. Estradiol inhibits osteoblast apoptosis via promotion of autophagy through the ER-ERK-mTOR pathway. Apoptosis. 2013;18:1363-75. doi:10.1007/s10495-013-0867-x.

28. Yang YH, Zheng XF, Li B, Jiang SD, Jiang LS. Increased activity of osteocyte autophagy in ovariectomized rats and its correlation with oxidative stress status and bone loss. Biochem Bioph Res Co. 2014;451:86-92. doi:10.1016/j.bbrc.2014.07.069.

29. Zhang XJ, Li LA, Chen S, Yang DH, Wang Y, Zhang X, Wang Z, Le WD. Rapamycin treatment augments motor neuron degeneration in SOD1(G93A) mouse model of amyotrophic lateral sclerosis. Autophagy. 2011;7:412-25. doi:10.4161/auto.7.4.14541.

\section{Figures}



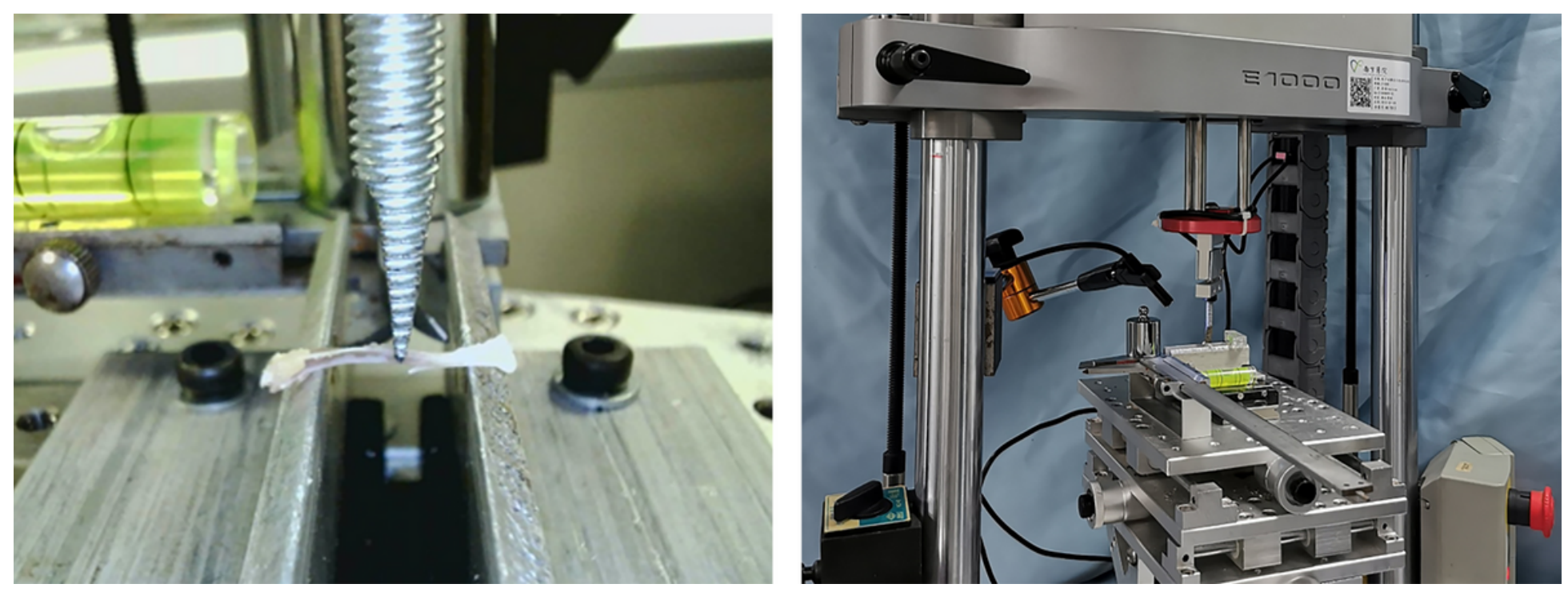

Figure 1

The three-point bending test set-up. The mouse tibia was placed between two supports with a span of 10 $\mathrm{mm}$ on a slider. A compressive force was applied to the bone until it was broken

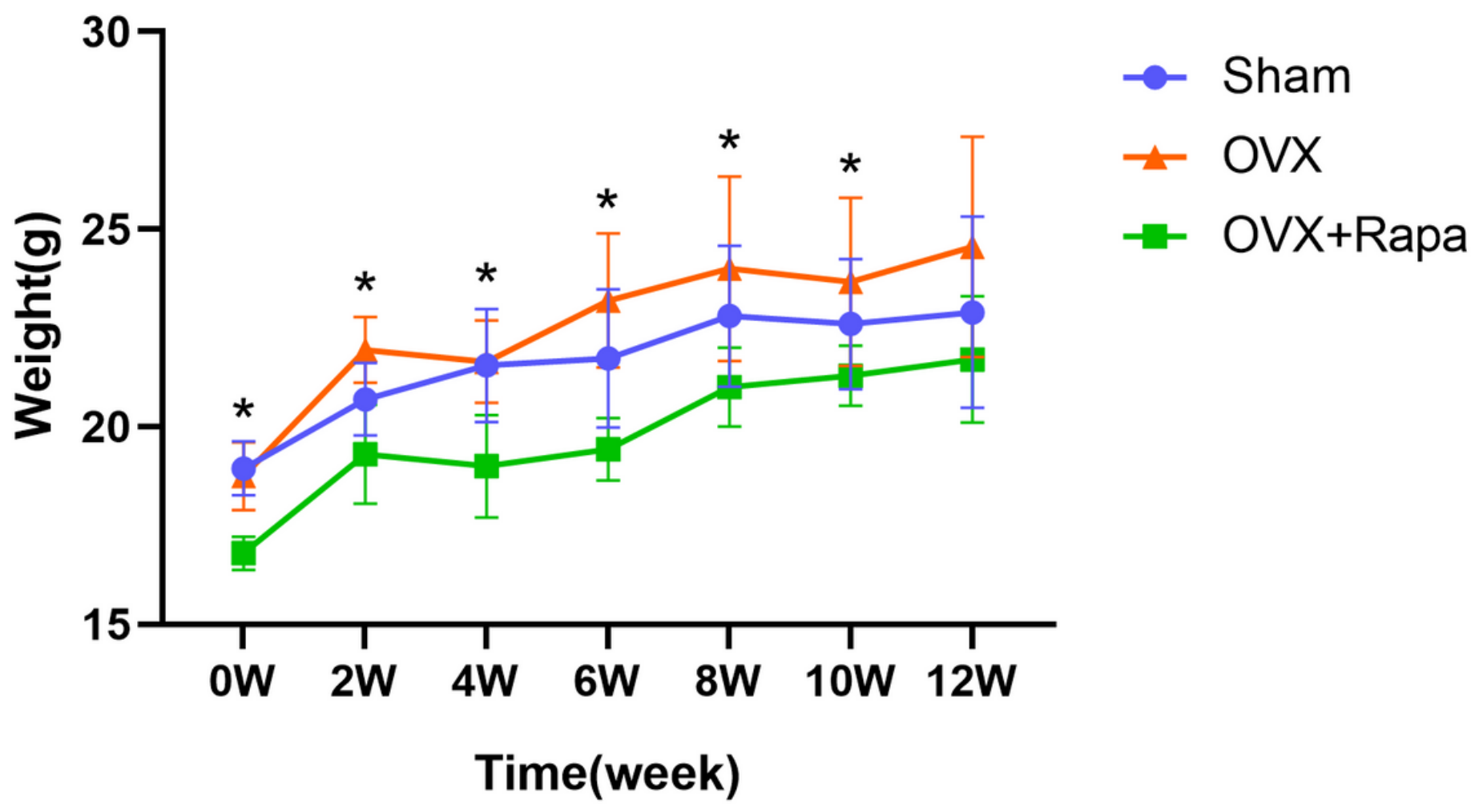

Figure 2

The body weight of mice during 12 weeks. ${ }^{*} \mathrm{P}<0.05$ 


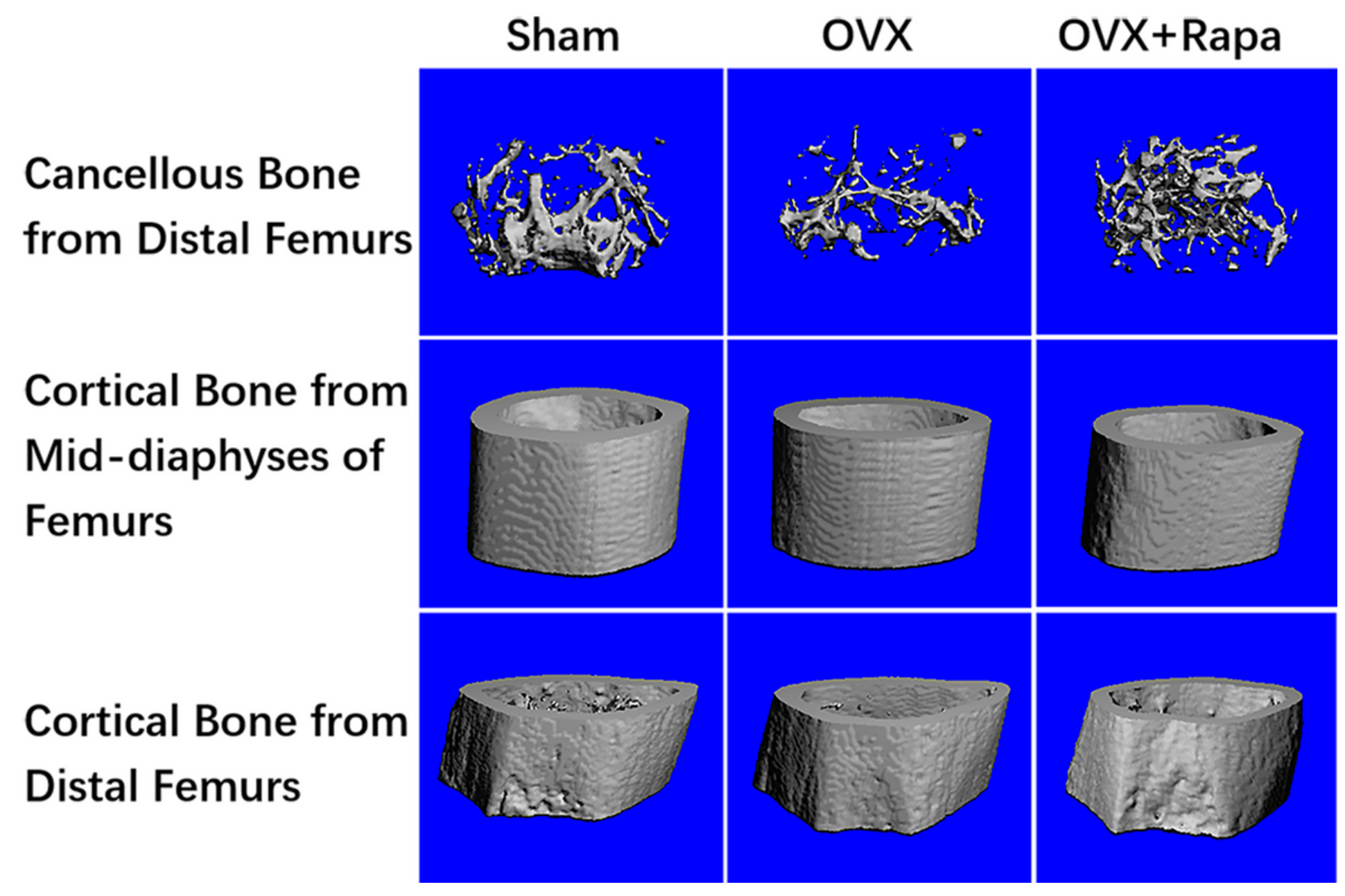

Figure 3

The micro-CT images of both cancellous bone and cortical bone of femurs at the distal and diaphyses 

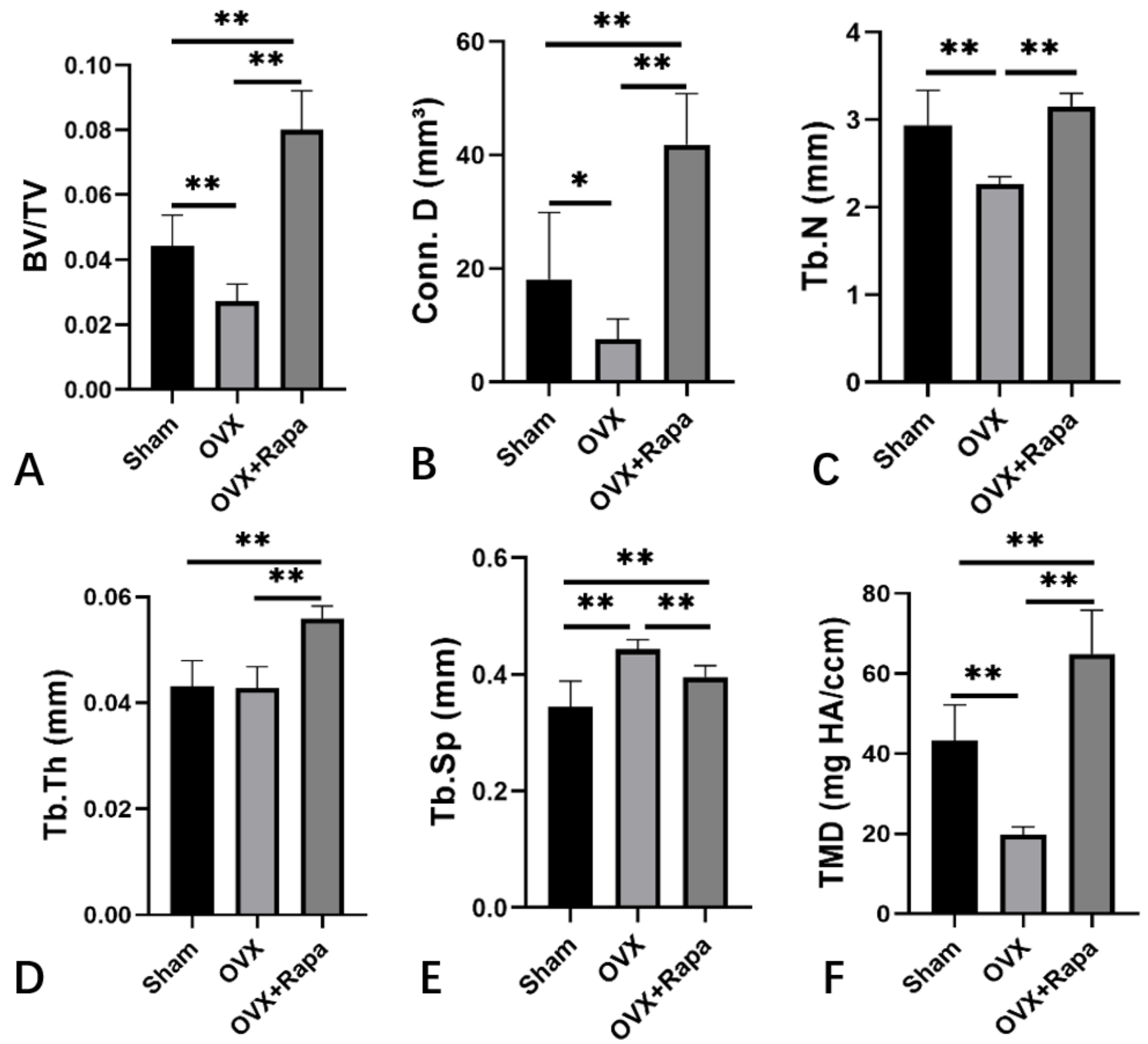

Figure 4

Micro-CT analysis of cancellous bone from distal femurs: (A) bone volume/tissue volume (BV/TV), (B) connection density of trabecular (Conn.D), (C)trabecular number (Tb.N), (D) trabecular thickness (Tb.Th), (E) trabecular separation (Tb.Sp), ( $F$ ) tissue mineral density (TMD). ${ }^{*}<<0.05$, ** $P<0.01$ 
Sham

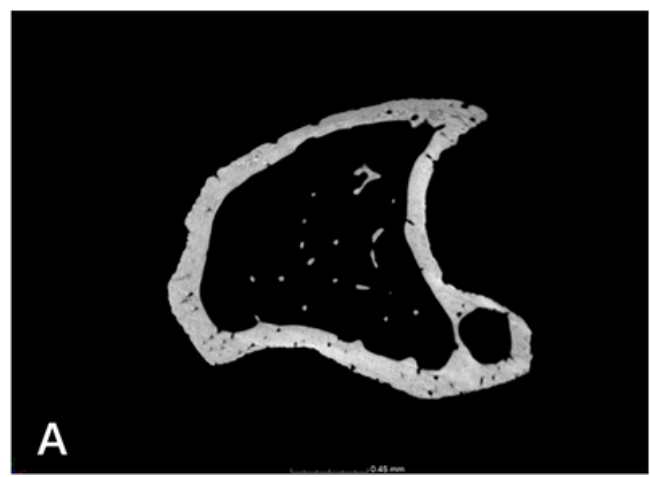

OVX

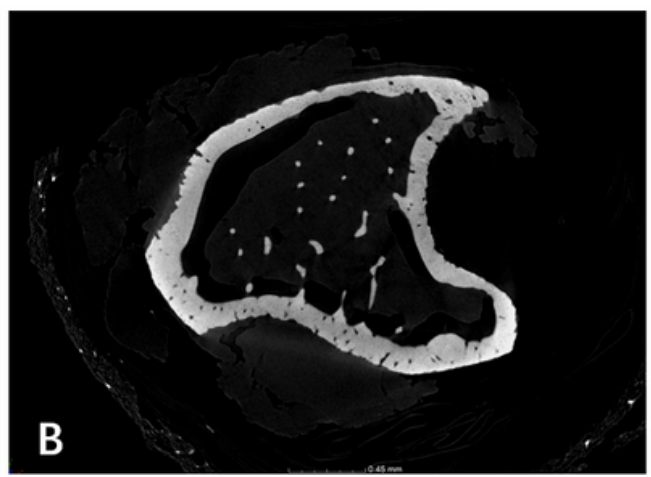

OVX+Rapa

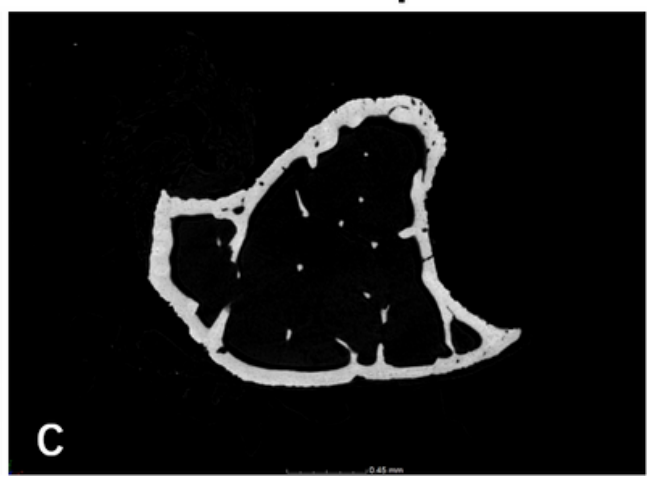

Figure 5

High-resolution micro-CT scanning analysis of the vascular canal porosity of the proximal tibial cortical bone

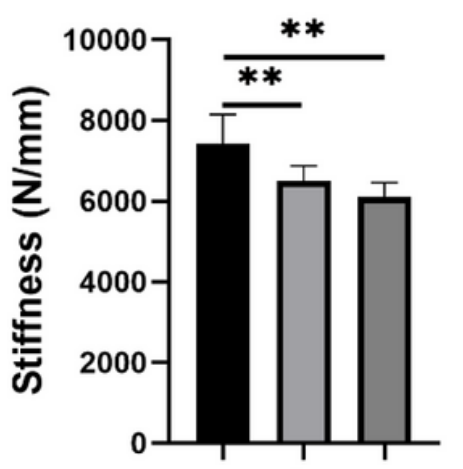

A

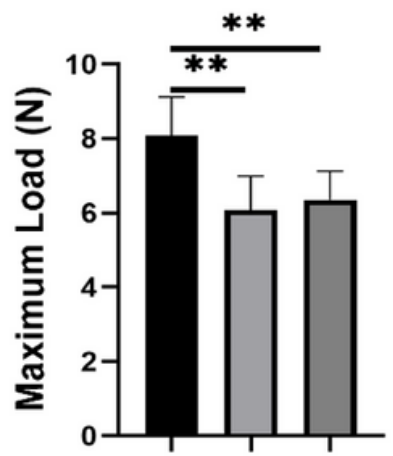

B
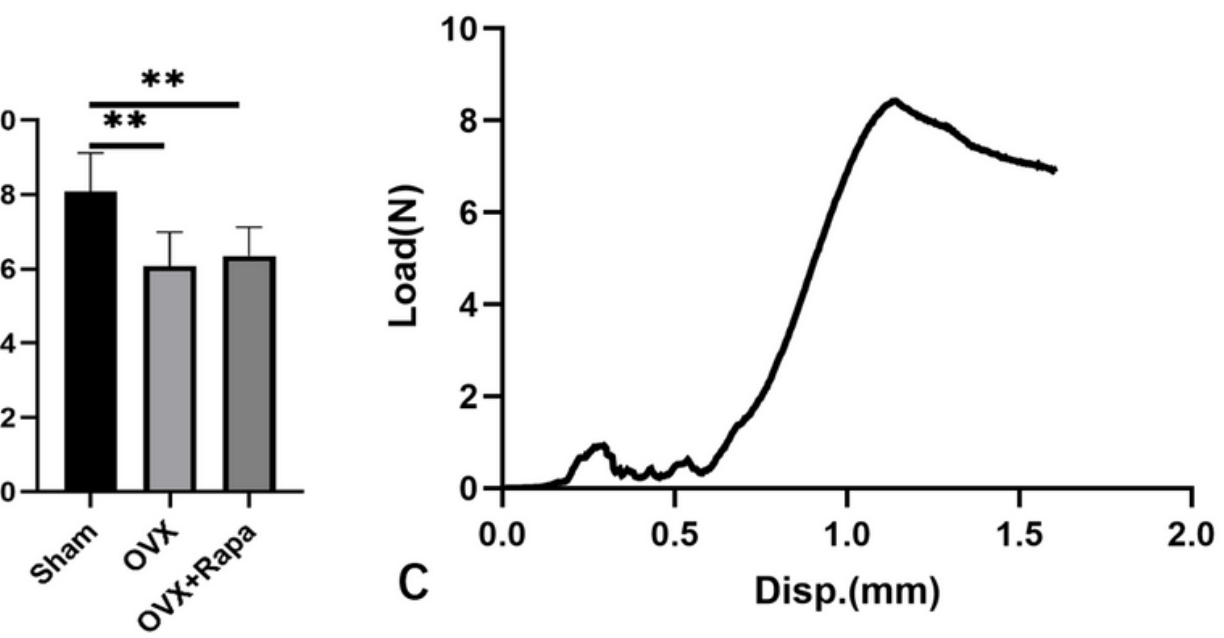

Figure 6

Tests of biomechanical properties: (A) stiffness $(\mathrm{N} / \mathrm{mm})$ evaluated by the simulated compressive test, (B) maximum load $(\mathrm{N})$ measured by the three-point bending test, $(\mathrm{C})$ one of the displacement-load curves from the three-point bending test. ** $\mathrm{P}<0.01$ 
Sham

a

HE

staining

TRAP

staining
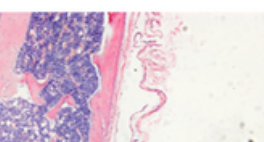

b

OVX

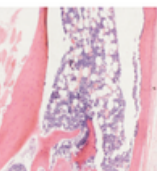

OVX+Rapa

C

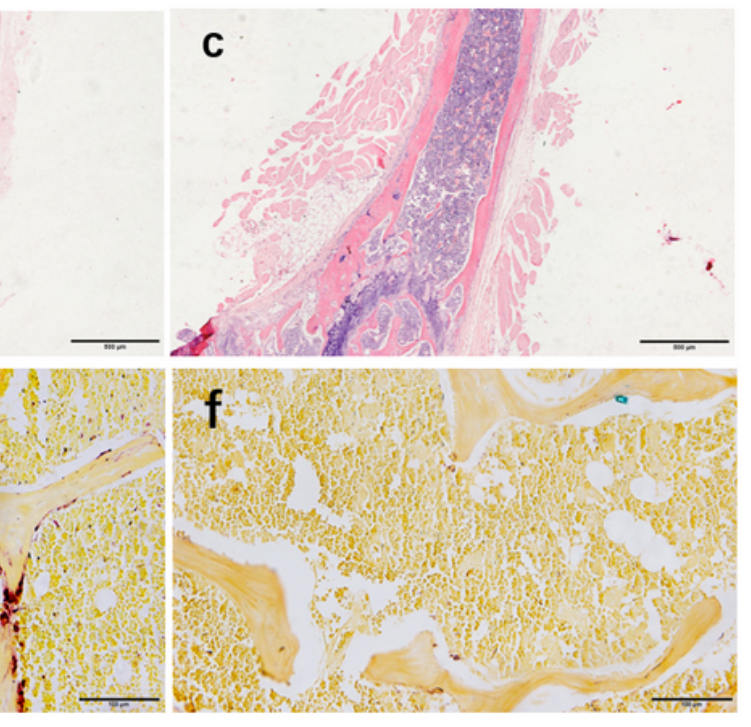

Figure 7

Histochemical Staining: (A-C) the HE staining, (D-F) the TRAP staining
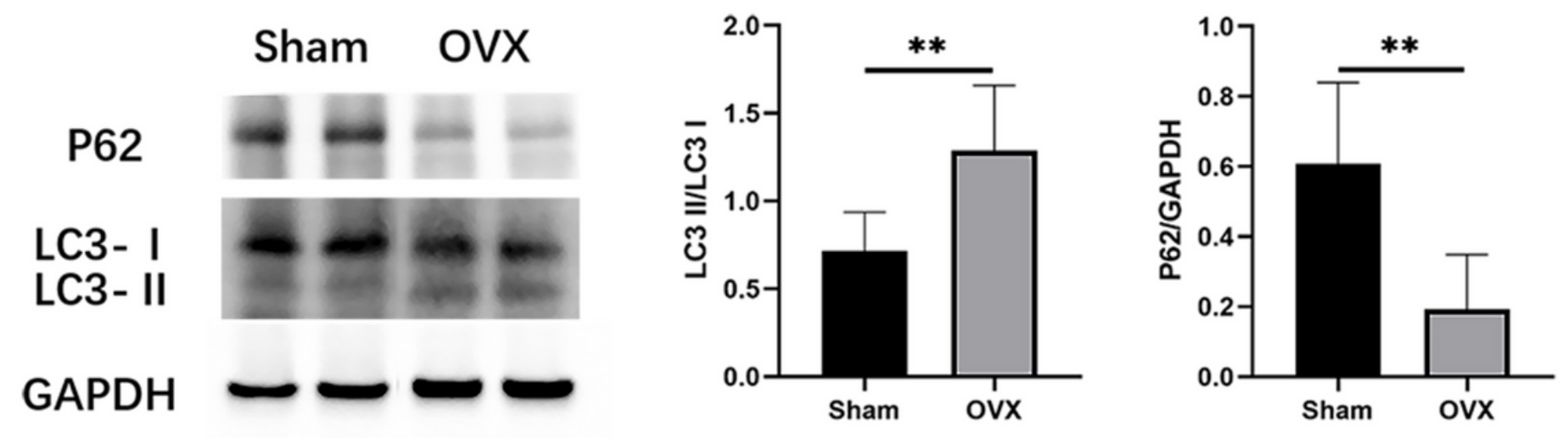

Figure 8

Western blot and qualification for LC3-『/LC3-『and P62. ** $P<0.01$ 